\title{
Alcances de la Confiabilidad en la Medición Antropométrica: Un Aporte para el Escalonamiento de la Formación Competente en Pregrado, Una Experiencia Piloto
}

\author{
Scope of Reliability in Anthropometric Measurement: A contribution for the \\ Staggering of the Undergraduate Competency Formation, a Pilot Experience
}

Rodrigo Muñoz Cofré ${ }^{1}$; Mariano del Sol${ }^{1}$; Francisca Villagrán Silva ${ }^{1}$;

Pablo A. Lizana ${ }^{2}$; Gabriel Nasri Marzuca-Nassr ${ }^{3}$ \& Máximo Escobar Cabello ${ }^{4}$

MUÑOZ, C. R.; DEL SOL, M.; VILLAGRÁN, S. F.; LIZANA, P. A.; MARZUCA-NASSR, G. N \& ESCOBAR, C. M. Alcances de la confiabilidad en la medición antropométrica: un aporte para el escalonamiento de la formación competente en pregrado, una experiencia piloto. Int. J. Morphol., 36(4):1298-1304, 2018.

RESUMEN: El objetivo de la presente investigación fue determinar el nivel de confiabilidad de las mediciones antropométricas relacionadas al tórax, realizadas por un estudiante de licenciatura en kinesiología (ELK) comparandolas con las de un antropometrista experto (AE). El proceso consistió en el escalonamiento de competencias dividido en tres etapas: i) desarrollo de competencias teóricas y prácticas, ii) Determinar idea de investigación y construcción de su marco teórico y iii) adquisición de confiabilidad, el ELK realizó este ejercicio en relación al AE International Society for the Advancement of Kinanthropometry (ISAK) II. Para este punto, se reclutaron seis participantes, los cuales fueron aleatorizados y medidos por ambos evaluadores. Se registraron los diámetros, perímetros y pliegues relacionados al tórax utilizando los protocolos de la ISAK, para posteriormente calcular el error técnico de la medición (ETM) y el coeficiente de correlación de concordancia de Lin (CCC). El ETM fue el aceptado para diámetros, perímetros y pliegues, salvo en el valor del pliegue bicipital, el que mostró un 5,60 \%. La fuerza de la relación fue moderada a sustancial en perímetros y pliegues y pobre en los diámetros entre el ELK y el AE. En conclusión, el ETM se encontró dentro de los rangos permitidos salvo el pliegue bicipital. Sin embargo, ELK mostró un nivel de concordancia moderado a sustancial en perímetros y pliegues y pobre en diámetros de tórax en relación al AE.

PALABRAS CLAVE: Competencia; Confiabilidad; Antropometría; Tórax.

\section{INTRODUCCIÓN}

Actualmente en el marco nacional de cualificaciones, Chile promueve la igualdad de oportunidades, facilitando el acceso de las personas a programas formativos de calidad con el propósito de tener el reconocimiento de las competencias adquiridas (Kri et al., 2016). Además, incorpora un conjunto de aspectos conceptuales y metodológicos que necesitan de la participación de educadores expertos en los distintos niveles para la definición de los mismos, y hacerlos explícitos; formalizar descriptores de niveles de aprendizaje; medición de la carga de trabajo relacionada con los sistemas de acumulación y transferencia de créditos; perfiles de las cualificaciones; y conexión con un sistema de aseguramiento de la calidad (Montt \& Rosso, 2014).
A ello se suma que en las recientes décadas los estudios de las diferentes disciplinas han experimentado significativos cambios alrededor del mundo tales como reducción en el número de créditos y horas de aprendizaje (Inzunza $e t$ al., 2007; Lizana et al., 2015). Por otra parte, en el contexto de la instrucción es posible utilizar protocolos, modelos, técnicas que en sus formas necesitan la precisión y el conocimiento que exige de parte del profesor, la certeza que la reproducibilidad de la técnica cuente con un sistema de control que habilite adecuadamente al estudiante en formación (Becerra et al., 2015). Complementariamente a esta temática, las reformas curriculares se han preocupado de reducir el número de horas presenciales en orden a concentrar programas

\footnotetext{
${ }^{1}$ Programa de Doctorado en Ciencias Morfológicas, Universidad de La Frontera, Temuco, Chile.

${ }^{2}$ Laboratory of Morphological Sciences, Instituto de Biología, Pontificia Universidad Católica de Valparaíso, Valparaíso, Chile.

${ }^{3}$ Departamento de Medicina Interna, Facultad de Medicina, Universidad de La Frontera, Temuco, Chile.

${ }^{4}$ Programa de Especialidad en Función y Disfunción Ventilatoria, Universidad Católica del Maule, Chile.
} 
MUÑOZ, C. R.; DEL SOL, M.; VILLAGRÁN, S. F.; LIZANA, P. A.; MARZUCA-NASSR, G. N \& ESCOBAR, C. M. Alcances de la confiabilidad en la medición antropométrica: un aporte para el escalonamiento de la formación competente en pregrado, una experiencia piloto. Int. J. Morphol., 36(4):1298-1304, 2018.

evidentemente abultados, como también estimular el aprendizaje independiente de las destrezas interpersonales.

Un caso particular alude a la enseñanza de las técnicas de medición antropométricas, las cuales han experimentado como otras la dificultad de enfrentar la demanda de un número creciente de estudiantes, lo que ha significado una modificación en la relación del número de estos por profesor y, por lo tanto, la restricción del acceso a la manipulación confiable de los procedimientos que requieren una baja tasa de error (Kouchi et al., 1999). Las consecuencias de las alteraciones en las medidas antropométricas debido a las variaciones inter-evaluadores son transversales y, pueden estar presentes en los resultados de las medidas hechas en el deporte, la ingeniería, el vestuario, las universidades y los centros de formación profesional (Perini, 2005). Como es sabido la antropometría corresponde a una serie de mediciones de técnicas sistematizadas que expresan cuantitativamente todas las dimensiones del cuerpo humano (Piñeda Geraldo, 2015). Los estudios de las medidas antropométricas se han incrementado en las últimas décadas centrando su objetivo en el error de medición, origen y magnitud, para llegar a sostener que el error de medición es un problema de alta relevancia en la antropometría (Norton \& Olds, 2004). Así, pequeñas modificaciones en la técnica de medición pueden traducir resultados significativamente alterados.

Asumiendo que el desarrollo tecnológico ha logrado disminuir la incertidumbre de los resultados, es la medición como fenómeno de aprendizaje, la que requiere refinar exhaustivamente la confiabilidad de sus acciones. Así la confiabilidad es el grado en que un dato está libre de error, para proporcionar estabilidad y precisión a las observaciones (Streiner, 2003). Esta se puede medir a través de la consistencia interna, la aplicación de un test-retest o la aplicación de la misma prueba a una persona, pero teniendo dos operadores distintos (confiabilidad inter-evaluador). Correlacionar los puntajes obtenidos entre dos evaluadores entonces permitirá observar hasta qué punto es posible corregir el factor azar (Alarcón \& Muñoz, 2008), complementariamente, es el error técnico de la medición (ETM), quién determina el estadístico de la calidad de la medida (Piñeda Geraldo).

En este escenario, un estudiante de licenciatura en kinesiología (ELK) junto con un adecuado escalonamiento de los momentos formativos, tiene que demostrar un manejo integral para obtener resultados de calidad en sus mediciones, pero además y, en un marco de formación por competencias, debe alcanzar altos niveles de reproducibilidad (Gouveia et al., 2014). Así para la actuación competente de ELK será necesario dedicar tiempos y espacios formativos a través de sistemas modulares, junto con disponer de un modelo de educación clínica individual con el propósito de que integre las habilidades básicas, los protocolos de las pruebas y el análisis basado en los resultados obtenidos (Knudson et al., 1976). A pesar del consenso de estas evidencias, se desconoce la especificidad de las condiciones que puedan garantizar el entrenamiento eficiente de un ELK, esto sin dejar de mantener las ya conocidas habilidades de la relación con el paciente y el propio compromiso con la prueba específica para que los resultados representen los valores reales del paciente (Rodríguez, 2015)

Otro punto fundamental de la formación por competencias es la solución de problemáticas reales de la población (Kri et al.), en este escenario, las enfermedades del sistema respiratorio a nivel local constituyen el tercer y segundo grupo de causas de muerte y egreso hospitalario en mayores de 65 años, respectivamente. Por lo que una correcta valoración del sistema respiratorio en su totalidad es de gran importancia (Olmos et al., 2015).

La hipótesis de la presente investigación es que la adquisición de confiabilidad en las mediciones antropométricas responde a una programación inducida de un periodo formativo escalonado para alcanzar la suficiencia investigativa y ésta será producto de la sumatoria de experiencias. En este escenario, el objetivo de la presente investigación fue determinar el nivel de confiabilidad de las mediciones antropométricas de un ELK al concluir su escalonamiento formativo, comparando sus resultados con un antropometrista experto (AE).

\section{MATERIAL Y MÉTODO}

El proceso de escalonamiento en la adquisición de la confiabilidad del ELK en la medición de antropometría de tórax, se dividió en tres etapas (Fig. 1); i) desarrollo de competencias teóricas y prácticas, aquí el estudiante cumplió con los requerimientos curriculares del módulo Interacción en Centros de Mediana Complejidad del segundo año de la carrera de Kinesiología del Universidad Católica del Maule (Proyecto Formativo Profesional de Pregrado de la Carrera de Licenciatura en Kinesiología, 2011), esto correspondió a 3 horas teóricas y 4 prácticas de la unidad de antropometría, equivalentes a la evaluación de 4 sujetos según normativa de International Society for the Advancement of Kinanthropometry (ISAK), ii) Determinar idea de investigación y construcción de su marco teórico, proceso que resultó en la selección de mediciones antropométricas de tórax, invirtiendo 36 horas y iii) adquisición de confiabilidad, el ELK realizó este ejercicio en relación a la AE ISAK II, recibió una instrucción teórica de 1 hora y evaluó las variables relacionadas al tórax (Fig. 2) en seis sujetos, mientras en paralelo el AE realizaba las misma mediciones, este pro- 


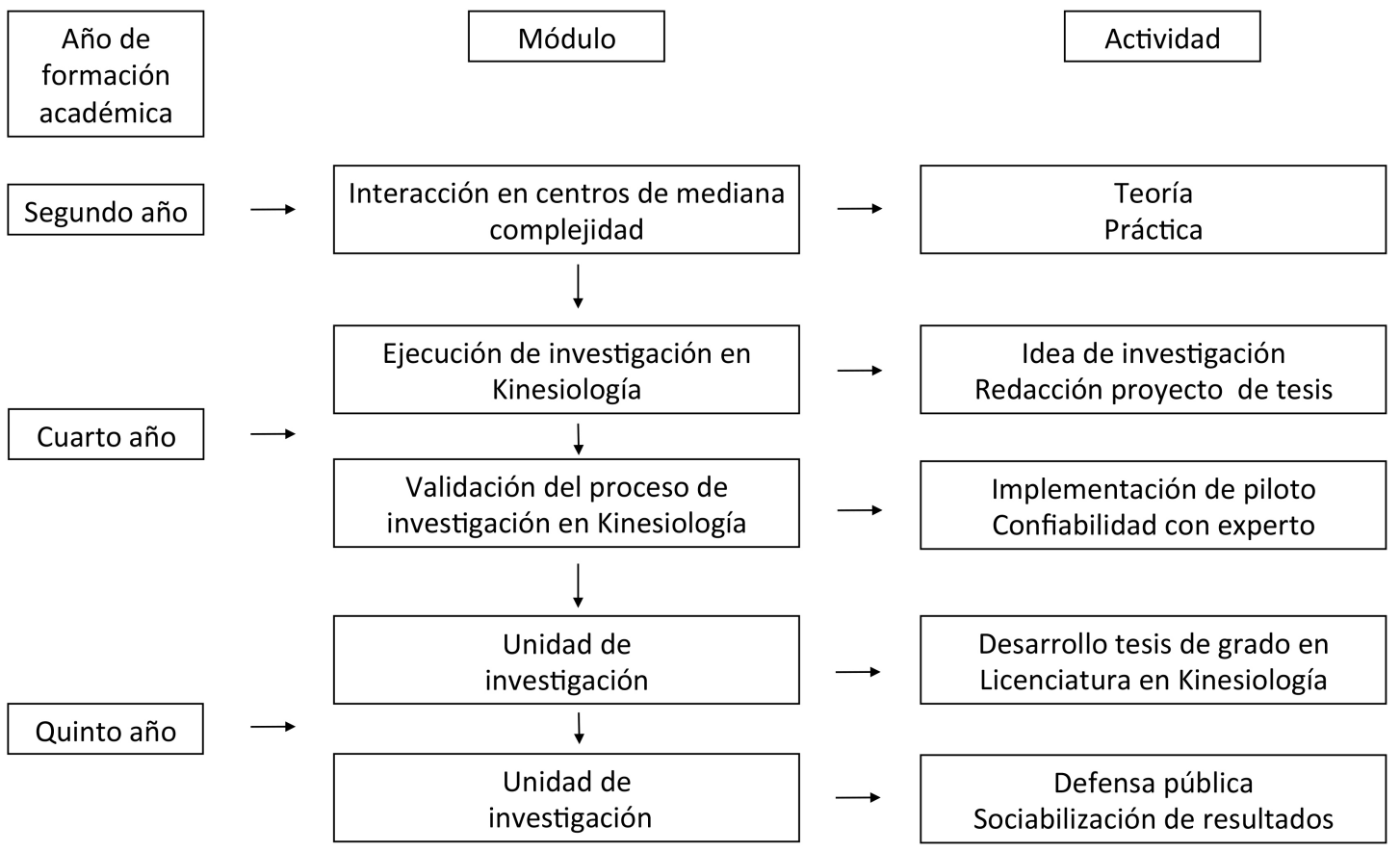

Fig. 1. Escalonamiento de la Formación Competente, integración del año de formación académica, módulo y actividad asociada

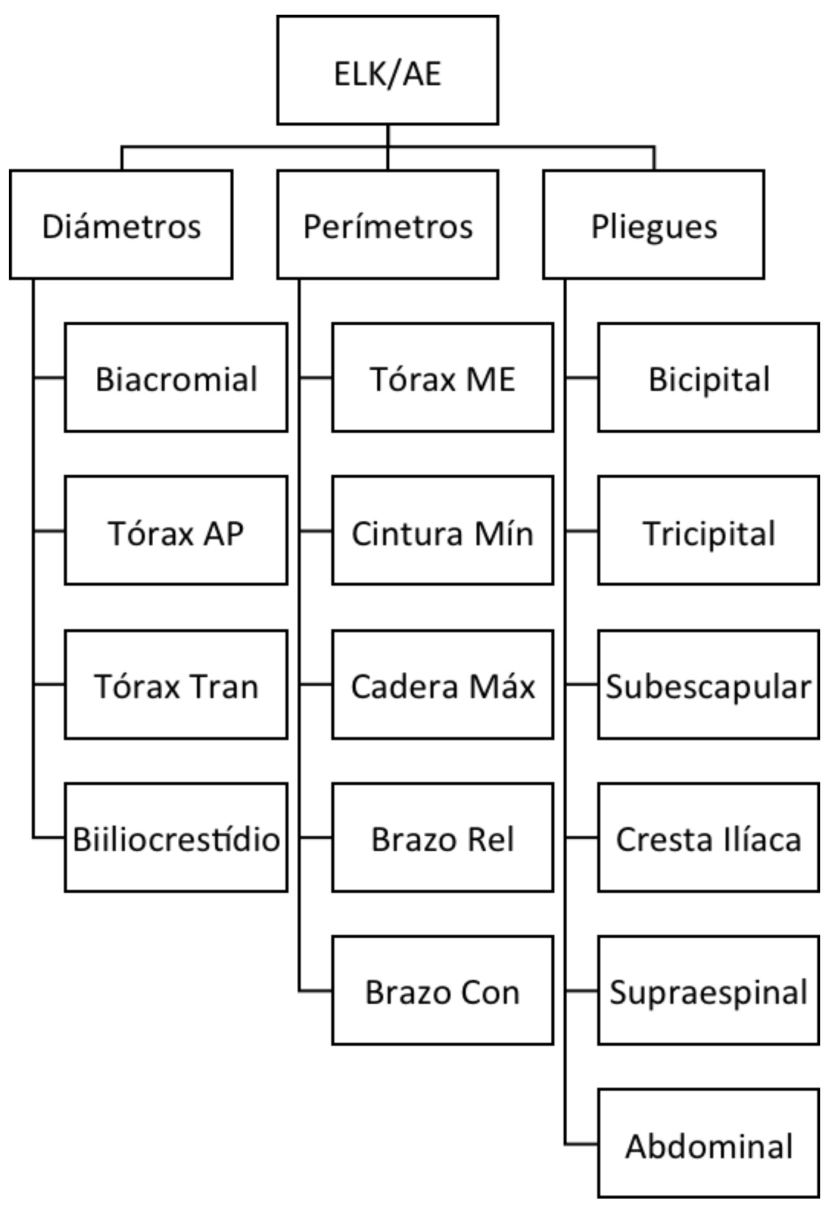

Fig. 2. Detalle de los diámetros, perímetros y pliegues evaluados. ELK: estudiante de licenciatura en kinesiología; AE: antropometrista experto; AP: antero posterior; Tran: transverso; ME: meso-esternal; Mín: mínima; Máx: máxima; Rel: relajado; Con: contraído 
MUÑOZ, C. R.; DEL SOL, M.; VILLAGRÁN, S. F.; LIZANA, P. A.; MARZUCA-NASSR, G. N \& ESCOBAR, C. M. Alcances de la confiabilidad en la medición antropométrica: un aporte para el escalonamiento de la formación competente en pregrado, una experiencia piloto. Int. J. Morphol., 36(4):1298-1304, 2018.

ceso tuvo 6 horas de dedicación. Los participantes fueron aleatorizados y medidos por ambos evaluadores para posteriormente realizar el análisis estadístico.

Diámetros, perímetros y pliegues: Se midieron con un antropómetro mayor Rosscraft (Campbell 20 ${ }^{\circledR}$, Richmond, Canadá), cinta antropométrica de metal Rosscraft y un caliper Rosscraft (SLIGUI Body Fat Caliper ${ }^{\circledR}$ Beta Technology, Richmond, Canadá), respectivamente. Los diámetros, perímetros y pliegues obtenidos se encuentran en la Figura 1, se utilizaron los protocolos de la ISAK (International Society for the Advancement of Kinanthropometry, 2001).

Análisis estadístico: El manejo descriptivo de las variables fue mediante el promedio, para determinar el error técnico de la medición (ETM) absoluto y relativo se utilizaron las siguientes fórmulas.

$\begin{array}{cc}\text { ETM Absoluto } & \text { ETM Relativo } \\ \frac{\sum \sqrt{ } d^{2}}{n} & \frac{\text { ETM }}{\text { VMV }} \times 100\end{array}$

$\Sigma$ : suma; $\sqrt{ } \mathrm{d}^{2}$ : raíz de la diferencia de las medidas al cuadrado; n: número de mediciones; VMV: valor medio de la variable.

Tabla I. Características antropométricas de los sujetos estudiados.

\begin{tabular}{lc}
\hline Variable & Valor \\
\hline Sexo $(\mathrm{H} / \mathrm{M})$ & $5 / 0$ \\
Edad $(\mathrm{años})$ & $25,80 \pm 5,97$ \\
Peso $(\mathrm{kg})$ & $73,45 \pm 9,82$ \\
Talla $(\mathrm{m})$ & $1,76 \pm 0,06$ \\
IMC $\left(\mathrm{kg} / \mathrm{m}^{2}\right)$ & $23,91 \pm 0,06$ \\
\hline
\end{tabular}

Los valores son presentados en promedio \pm una desviación estándar. $\mathrm{H}$, hombre; $\mathrm{M}$, mujer; IMC, índice de masa corporal.
Posteriormente, a través del programa estadístico GraphPad Prism (versión 5.0 ${ }^{\circledR}$, San Diego, USA), se estableció la correlación para diámetros, perímetros y pliegues entre el valor obtenido por el estudiante y el experto. Para evaluar la concordancia de las mediciones entre el estudiante y el experto se ocupó el coeficiente de correlación de concordancia de Lin (CCC) (Lin, 1989 \& Lin, 2000), estos resultados se categorizaron según la escala descriptiva para variables continuas de McBride (2005), la cual se estructura de la siguiente manera, un valor $>0,99$ : relación casi perfecta; 0,95-0,99: relación sustancial; 0,90-0,95: relación moderada y $<0,90$ : relación pobre. Por último el nivel de significancia estadística se estableció en un $\mathrm{p}<0,05$.

\section{RESULTADOS}

La muestra correspondió a cinco sujetos de sexo mas-

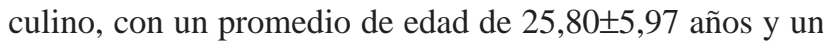
índice de masa corporal (IMC) de $23,91 \pm 0,06 \mathrm{~kg} / \mathrm{m}^{2}$ (Tabla I). El ETM se encontró dentro de la tolerancia permitida (Tablas II, III y IV) en la mayoría de las mediciones de diámetros, perímetros y pliegues, salvo en el valor del pliegue bicipital, el que mostró un 5,60 \% (Tabla IV). Además, la fuerza de la relación entre el AE y el ELK fue pobre para todos los diámetros (Tabla II). Por otra parte, en los perímetros todos los resultados mostraron una relación moderada a sustancial entre el AE y el ELK, sin embargo, destaca el perímetro de tórax meso-esternal con un CCC de 0,891, el cual tuvo una relación pobre (Tabla III). Del mismo modo, los pliegues tuvieron una relación moderada a sustancial entre el AE y el ELK, pese a esto, el pliegue tricipital mostró una relación pobre con un CCC de 0,833 entre ambos (Tabla IV).

Tabla II. Error técnico de la medición en diámetros entre estudiante y experto

\begin{tabular}{|c|c|c|c|c|c|c|c|c|}
\hline Variable & Promedio & $\mathrm{CCC}$ & Diferencia & IC $95 \%$ & $\begin{array}{c}\text { ETM } \\
\text { (absoluto) }\end{array}$ & VMV & $\begin{array}{c}\text { ETM } \\
\text { (relativo, \%) }\end{array}$ & $\begin{array}{c}\text { Tolerancia } \\
\text { permitida }\end{array}$ \\
\hline \multicolumn{9}{|l|}{ Biacromial } \\
\hline $\mathrm{AE}$ & 39,18 & & & & & & & \\
\hline ELK & 38,94 & 0,786 & 0,040 & $0,401-1,172$ & 0,19 & 39,23 & 0,50 & $1-2 \mathrm{~mm}$ \\
\hline \multicolumn{9}{|l|}{ Tórax AP } \\
\hline $\mathrm{AE}$ & 37,33 & & & & & & & \\
\hline ELK & 36,78 & $-0,227$ & $-1,220$ & $-0,973-0,520$ & 0,28 & 37,06 & 0,76 & $1-2 \mathrm{~mm}$ \\
\hline \multicolumn{9}{|l|}{ Tórax Tran } \\
\hline $\mathrm{AE}$ & 27,80 & & & & & & & \\
\hline ELK & 27,82 & 0,518 & $-0,380$ & $-0,264-1,300$ & 0,43 & 27,81 & 1,50 & $2-3 \mathrm{~mm}$ \\
\hline \multicolumn{9}{|c|}{ Bi-ilicrestídio } \\
\hline $\mathrm{AE}$ & 26,33 & & & & & & & \\
\hline ELK & 25,67 & 0,681 & $-0,360$ & $0,369-0,992$ & 0,39 & 26,00 & 1,50 & $1-2 \mathrm{~mm}$ \\
\hline
\end{tabular}

CCC: coeficiente de correlación de concordancia de Lin; IC: intervalo de confianza; ETM: error técnico de la medición; VMV: valor medio de la variable; AE: antropometrista experto; ELK: estudiante de licenciatura en kinesiología; AP: antero-posterior; Tran: transverso; mm: milímetros. 
MUÑOZ, C. R.; DEL SOL, M.; VILLAGRÁN, S. F.; LIZANA, P. A.; MARZUCA-NASSR, G. N \& ESCOBAR, C. M. Alcances de la confiabilidad en la medición antropométrica: un aporte para el escalonamiento de la formación competente en pregrado, una experiencia piloto. Int. J. Morphol., 36(4):1298-1304, 2018.

Tabla III. Error técnico de la medición en perímetros entre estudiante y experto

\begin{tabular}{|c|c|c|c|c|c|c|c|c|}
\hline Variable & Promedio & $\mathrm{CCC}$ & Diferencia & IC 95\% & $\begin{array}{c}\text { ETM } \\
\text { (absoluto) }\end{array}$ & VMV & $\begin{array}{c}\text { ETM } \\
\text { (rela tivo, \%) }\end{array}$ & $\begin{array}{l}\text { Tolerancia } \\
\text { permitida }\end{array}$ \\
\hline \multicolumn{9}{|l|}{ Tórax ME } \\
\hline $\mathrm{AE}$ & 99,01 & & & & & & & \\
\hline ELK & 97,85 & 0,891 & 0,820 & $0,687-1,096$ & 0,88 & 98,43 & 0,89 & $1-2 \%$ \\
\hline \multicolumn{9}{|c|}{ Cintura Min } \\
\hline $\mathrm{AE}$ & 81,60 & & & & & & & \\
\hline ELK & 81,44 & 0,968 & $-0,900$ & $0,900-1,036$ & 0,53 & 81,52 & 0,65 & $2-3 \%$ \\
\hline \multicolumn{9}{|c|}{ Cadera Máx } \\
\hline $\mathrm{AE}$ & 99,92 & & & & & & & \\
\hline ELK & 98,72 & 0,974 & 0,400 & $0,926-1,022$ & 0,68 & 99,32 & 0,68 & $2-3 \%$ \\
\hline \multicolumn{9}{|l|}{ Brazo Rel } \\
\hline $\mathrm{AE}$ & 30,18 & & & & & & & \\
\hline ELK & 30,65 & 0,960 & $-0,620$ & $0,889-1,030$ & 0,26 & 30,42 & 0,85 & $2 \mathrm{~mm}$ \\
\hline \multicolumn{9}{|c|}{ Brazo Con } \\
\hline $\mathrm{AE}$ & 31,57 & & & & & & & \\
\hline ELK & 32,20 & 0,926 & $-0,700$ & $0,789-1,063$ & 0,32 & 31,88 & 1,00 & $2 \mathrm{~mm}$ \\
\hline
\end{tabular}

CCC: coeficiente de correlación de concordancia de Lin; IC: intervalo de confianza; ETM: error técnico de la medición; VMV: valor medio de la variable; ME: mesoesternal; AE: antropometrista experto; ELK: estudiante de licenciatura en kinesiología; Mín: mínima; Máx: máxima; Rel: relajado; Con: contraído; mm: milímetros.

Tabla IV. Error técnico de la medición en pliegues cutáneos entre estudiante y experto.

\begin{tabular}{|c|c|c|c|c|c|c|c|c|}
\hline Variable & Promedio & $\mathrm{CCC}$ & Diferencia & IC 95\% & $\begin{array}{c}\text { ETM } \\
\text { (absoluto) }\end{array}$ & VMV & $\begin{array}{c}\text { ETM } \\
\text { (relativo, \%) }\end{array}$ & $\begin{array}{l}\text { Tolerancia } \\
\text { permitida }\end{array}$ \\
\hline \multicolumn{9}{|l|}{ Bicipital } \\
\hline $\mathrm{AE}$ & 5,33 & & & & & & & \\
\hline ELK & 5,25 & 0,938 & $-0,200$ & $0,859-1,016$ & 0,30 & 5,29 & 5,60 & $5 \%$ \\
\hline \multicolumn{9}{|l|}{ Tricipital } \\
\hline $\mathrm{AE}$ & 10,83 & & & & & & & \\
\hline ELK & 10,92 & 0,833 & $-0,800$ & $0,531-1,136$ & 0,41 & 10,87 & 3,77 & $5 \%$ \\
\hline \multicolumn{9}{|c|}{ Subescapular } \\
\hline $\mathrm{AE}$ & 13,80 & & & & & & & \\
\hline ELK & 14,00 & 0,979 & $-0,400$ & $0,935-1,023$ & 0,18 & 13,55 & 1,32 & $5 \%$ \\
\hline \multicolumn{9}{|c|}{ Cresta Ilíaca } \\
\hline $\mathrm{AE}$ & 18,33 & & & & & & & \\
\hline ELK & 17,75 & 0,945 & 0,000 & $0,832-1,057$ & 0,76 & 18,04 & 4,21 & $5 \%$ \\
\hline \multicolumn{9}{|c|}{ Supraespinal } \\
\hline $\mathrm{AE}$ & 13,83 & & & & & & & \\
\hline ELK & 13,92 & 0,971 & $-0,600$ & $0,916-1,026$ & 0,22 & 13,88 & 1,58 & $5 \%$ \\
\hline \multicolumn{9}{|c|}{ Abdominal } \\
\hline $\mathrm{AE}$ & 18,67 & & & & & & & \\
\hline ELK & 17,25 & 0,966 & 0,800 & $0,896-1,037$ & 0,67 & 17,96 & 3,73 & $5 \%$ \\
\hline
\end{tabular}

CCC: coeficiente de correlación de concordancia de Lin; IC: intervalo de confianza; ETM: error técnico de la medición; VMV: valor medio de la variable; AE: antropometrista experto; ELK: estudiante de licenciatura en kinesiología.

\section{DISCUSIÓN}

El objetivo de la presente investigación fue determinar el nivel de confiabilidad de las mediciones antropométricas de un ELK y un AE. En este contexto, uno de los principales hallazgos fue un ETM mayor a la tolerancia permitida para el pliegue bicipital, además la fuerza de la relación fue pobre en todas las mediciones de diámetro, en perímetro de tórax mesoesternal y el pliegue de tríceps entre el ELK y el AE. Aquí la interacción direc- 
MUÑOZ, C. R.; DEL SOL, M.; VILLAGRÁN, S. F.; LIZANA, P. A.; MARZUCA-NASSR, G. N \& ESCOBAR, C. M. Alcances de la confiabilidad en la medición antropométrica: un aporte para el escalonamiento de la formación competente en pregrado, una experiencia piloto. Int. J. Morphol., 36(4):1298-1304, 2018.

ta entre experto y aprendiz en condiciones reales, demostró ser suficiente para la adquisición de competencias en la medición de perímetros y pliegues. Se confirma, que pese a la expansión actual de las herramientas tecnológicas (CD-ROMs, redes de Internet y multimedias) y las oportunidades de aprendizaje que generan, estas superen los clásicos tutoriales uno a uno (Farah \& Maybury, 2009).

Perini et al., con el propósito de estudiar el ETM interevaluador entre distintos antropometristas, realizaron mediciones de pliegues cutáneos en nueve diferentes puntos de 35 voluntarios. Específicamente el pliegue bicipital mostró un ETM de 5,8 \%. Por otra parte, Vegelin et al. (2003) con el objetivo de determinar el impacto del conocimiento técnico y experiencia del evaluador sobre la medición del pliegue tricipital, compararon el rendimiento de múltiples evaluadores con distinto nivel de expertiz, sus resultados indicaron que a mayor experiencia los resultados eran más precisos y exactos, además se confirmó la dificultad que existe en la medición de este pliegue (Vegelin et al.), estos resultado coincide con los de la presente investigación, tanto en bíceps como en tríceps, esto se debería; i) al mismo rango etario de la muestra, ii) a la experiencia, está descrito que la medición de pliegues depende en gran medida de la experiencia del evaluador (Loenneke et al., 2013), iii) la calibración del cáliper; debe realizarse anualmente y la precisión en la separación entre las garras debe comprobarse cada seis meses (Marks et al., 1989), iv) margen de error en el punto de medición, se sabe que diferencias de un milimetros en valores de pliegues cutáneos son significativamente diferentes (Hume \& Marfell-Jones, 2008), en este aspecto es importante destacar que a mayor grosor del pliegue existe mayor posibilidad de error en la medición (Marks et al.).

En detalle el ELK mostró una concordancia pobre en todas las mediciones de diámetro (Tabla II). Esto contrasta con los resultados encontrados por Piñeda Geraldo en el 2015, quien afirmó que las mediciones de diámetro tienen una alta confiabilidad, debido a que se realizan sobre puntos anatómicos óseos (Piñeda Geraldo). En el caso de las mediciones de tórax, tanto diámetros como perímetros, los puntos óseos son móviles debido al proceso de ventilación y a la imposibilidad de visualizar los volúmenes y capacidades pulmonares al momento de la evaluación, en este contexto, se ha documentado que los evaluadores expertos proporcionan instrucciones inequívocas y manejan de mejor manera a los pacientes/usuarios permitiéndoles así, concentrarse en generar un esfuerzo respiratorio acorde a lo que se necesita medir (Ruppel \& Enright, 2012). Por otra parte, en el caso de las medidas de diámetro biacromial y bi-iliocrestídeo, el manejo del malestar provocado por el posicionamiento de las púas del antropómetro sería mejor por parte del AE, así en ambos escenarios la experiencia en las mediciones sobre el tórax, marcaría una diferencia entre experto y aprendiz (Vegelin et al.). Además de esto, la exactitud de las medidas antropométricas, dependen de la minimización del error sistemático de los equipos y/o del antropometrista es fundamental, pese a esto y tomando las medidas correspondientes, el error no sistemático relacionado a estas dos instancias no se puede controlar (Marks et al.).

Como el proceso de aprendizaje de las técnicas antropométricas está basado en la identificación de puntos de referencias anatómicas estructuralmente definidos, los estudiantes aprenden a reconocerlos y, a la vez van censando los posibles errores de medición. Es aquí donde la interacción con el profesor para utilizar "tips" representa la clave entre un procedimiento de alto estándar o uno errático (Esparza, 1993). Por tanto, si consideramos la tensión reformista que enfrenta la formación de profesionales que se desenvuelven con estándares asociados al marco nacional de cualificaciones, junto con dar cuenta de procesos de formación competente y particularmente de aquellos que deben demostrar suficiencia investigativa. Es imprescindible que las modificaciones curriculares consideren progresivamente la incorporación de estrategias de certificación de habilitaciones de manera pedagógica, didáctica y competente.

Entre las limitaciones relevantes del presente estudio se encuentra el número reducido de sujetos evaluados, sin embargo, también es prudente destacar que este proceso se enmarca dentro de un escalonamiento progresivo de adquisición de habilidades resolutivas en condición real de una matriz basada en competencias (Proyecto Formativo Profesional de Pregrado de la Carrera de Licenciatura en Kinesiología). Además de esto, nos encontramos sobre una muestra seleccionada, lo cual responde al aumento progresivo de la complejidad a la cual fue sometido el aprendiz, aquí es importante un inicio con conocimiento y habilidades básicas las que se potenciarán a medida que vaya reflexionando sobre sus acciones diarias (Wainwright et al., 2010).

En conclusión, el ETM se encontró dentro de los rangos permitidos salvo el pliegue bicipital. Sin embargo, ELK mostró un nivel de concordancia moderado a sustancial en perímetros y pliegues y pobre en diámetros de tórax en relación al AE. La evidencia indica que es parte de la formación, por lo que lo recomendable es acompañar al estudiante en su reflexión en torno al proceso valorativo completo (Schön, 2010). Dado que por definición consensuada la competencia es una actuación profesional responsable en un contexto de incertidumbre. 
AGRADECIMIENTOS: A Daniel Donoso Riveros, Sebastián Rodríguez Valdés y Erick Sánchez Peña por su desinteresada colaboración.

MUÑOZ, C. R.; DEL SOL, M.; VILLAGRÁN, S. F.; LIZANA, P. A.; NASRI, M. G. \& ESCOBAR, C. M. Scope of reliability in anthropometric measurement: A contribution for the staggering of the undergraduate competency formation, a pilot experience. Int. J. Morphol., 36(4):1298-1304, 2018.

SUMMARY: The aim of the present investigation was to determine the reliability level of the anthropometric measurements related to the thorax performed by an undergraduate student in kinesiology (ELK) comparing them with those of an expert anthropometrist (AE). The process consisted of the staging of competencies divided into three stages: i) development of theoretical and practical skills, ii) Determine the idea of research and construction of its theoretical framework and iii) acquisition of reliability, the ELK carried out this exercise in relation to the AE International Society for the Advancement of Kinanthropometry (ISAK) II. For this point, five participants were recruited, which were randomized and measured by both evaluators. The diameters, perimeters and folds related to the thorax were recorded using the ISAK protocols, to calculate the technical error of the measurement (ETM) and the correlation coefficient of Lin's concordance (CCC). The ETM was accepted for diameters, perimeters and folds, except for the bicipital fold value, which showed a $5.60 \%$. The relationship strength was moderate to substantial in perimeters and folds and poor in the diameters between the ELK and the AE. In conclusion, the ETM was found within the permitted ranges except for the bicipital fold. However, ELK showed a moderate to substantial level of agreement in perimeters and folds and poor in chest diameters in relation to $\mathrm{AE}$.

Thorax.

KEY WORDS: Competence; Reliability; Anthropometry;

\section{REFERENCIAS BIBLIOGRÁFICAS}

Alarcón, A. M. \& Muñoz, N. S. Medición en salud: Algunas consideraciones metodológicas. Rev. Med. Chile, 136(1):125-30, 2008.

Becerra, D.; Grob, M.; Rodríguez, A.; Barker, M. J.; Consiglieri, L.; Ferri, G. \& Sabag, N. Academicachievement and perception of two teaching methods in histology: light microscopy and digital systems. Int. J. Morphol., 33(3):811-6, 2015

Esparza, F. Manual de Cineantropometría. Madrid, FEMEDE, 1993.

Farah, C. S. \& Maybury, T. Implementing digital technology to enhance student learning of pathology. Eur. J. Dent. Educ., 13(3):172-8, 2009.

Gouveia, V. H. O.; Araújo, A. G. F.; Maciel, S. S.; Ferreira, J. J. A. \& Santos, H. H. Reliability of the measures inter and intra-evaluators with universal goniometer and fleximeter. Fisioter. Pesqui., 21(3):229-35, 2014.

Hume, P. \& Marfell-Jones, M. The importance of accurate site location for skinfold measurement. J. Sports Sci., 26(12):1333-40, 2008.

International Society for the Advancement of Kinanthropometry (ISAK). International Standards for Anthroponetric Assessement. Potchefstroom, International Society for the Advancement of Kinanthropometry, 2001.

Inzunza, O.; Vargas, A. \& Bravo, H. Anatomía y neuroanatomía, disciplinas perjudicadas por la reforma curricular. Int. J. Morphol., 25(4):825-30, 2007.

Knudson, R. J.; Slatin, R. C.; Lebowitz, M. D. \& Burrows, B. The maximal expiratory flow-volume curve. Normal standards, variability, and effects of age. Am. Rev. Respir. Dis., 113(5):587-600, 1976.

Kouchi, M.; Mochimaru, M.; Tsuzuki, K. \& Yokoi, T. Interobserver errors in anthropometry. J. Hum. Ergol. (Tokyo), 28(1-2):15-24, 1999.
Kri, A. F.; Marchant, M. E.; Lazo, L. M. \& Cruz, F. S. Marco Nacional de Cualificaciones para la Educación Superior. Santiago de Chile, Ministerio de Educación, 2016. Disponible en: http://acreditaci.cl/wp-content/ uploads/2017/06/MNC.pdf

Lin, L. I. A concordance correlation coefficient to evaluate reproducibility. Biometrics, 45(1):255-68, 1989

Lin, L. I. A note on the concordance correlation coefficient. Biometrics, 56(1):324-5, 2000

Lizana, P. A.; Merino, C.; Bassaber, A.; Henríquez, R.; Vega-Fernández, G. \& Binvignat, $\mathrm{O}$. Learning human anatomy using three-dimensional models made from real-scale bone pieces: experience with the knee joint among pre-service biology teachers. Int. J. Morphol., 33(4):1299-306, 2015.

Loenneke, J. P.; Barnes, J. T.; Wilson, J. M.; Lowery, R. P.; Isaacs, M. N. \& Pujol, T. J. Reliability of field methods for estimating body fat. Clin. Physiol. Funct. Imaging, 33(5):405-8, 2013.

Marks, G. C.; Habicht, J. P. \& Mueller, W. H. Reliability, dependability, and precision of anthropometric measurements. The Second National Health and Nutrition Examination Survey 1976-1980. Am. J. Epidemiol., 130(3):578-87, 1989.

McBride, G. B. A proposal for Strength for Agreement Criteria of Lin Concordance Correlation Coefficient. Hamilton, National Institute of Water Atmospheric Research Ltda., 2005.

Montt, P. \& Rosso, P. P. Hacia un Marco Nacional de Cualificaciones para Chile. Santiago de Chile, Consejo Nacional de Educación, 2014. Disponible en: https://www.cned.cl/publicacion/hacia-un-marco-nacional-decualificaciones-para-chile

Norton, K. \& Olds, T. Antropométrica. Buenos Aires, Biossystem, 2004.

Olmos, C.; Mancilla, P.; Martínez, L. \& Astudillo, P. Epidemiología de las consultas respiratorias de adultos en Santiago de Chile desde 2003 a 2008. Rev. Med. Chile, 143(1):30-8, 2015.

Perini, T. A.; Oliveira, G. L.; Ornellas, J. S. \& Oliveira, F. P. Technical error of measurement in anthropometry. Rev. Bras. Med. Esporte, 11(1):81-5, 2005.

Piñeda Geraldo, A. Confiabilidad de medidas antropométricas en un grupo de estudiantes universitarias de Bogotá. Rev. Ing. Mat. Cienc. Inf., 1(2):29$48,2015$.

Proyecto Formativo Profesional de Pregrado de la Carrera de Licenciatura en Kinesiología. Talca, Dirección de Docencia, Vicerrectoría Académica, Universidad Católica del Maule, 2011.

Rodríguez, N. I. Confiabilidad de la fuerza muscular respiratoria y flujos espiratorios forzados en adolescentes sanos. Rev. Chil. Enferm. Respir., 31(2):86-93, 2015

Ruppel, G. L. \& Enright, P. L. Pulmonary function testing. Respir. Care, 57(1):165-75, 2012

Schön, D. La Formación de Profesionales Reflexivos: Hacia un Nuevo Diseño de la Enseñanza y el Aprendizaje en las Profesiones. $4^{\mathrm{a}}$ ed. Madrid, Paidós/ MEC, 2010.

Streiner, D. L. Starting at the beginning: an introduction to coefficient alpha and internal consistency. J. Pers. Assess., 80(1):99-103, 2003.

Vegelin, A. L.; Brukx, L. J.; Waelkens, J. J. \& Van den Broeck, J. Influence of knowledge, training and experience of observers on the reliability of anthropometric measurements in children. Ann. Hum. Biol., 30(1):65-79, 2003.

Wainwright, S. F.; Shepard, K. F.; Harman, L. B. \& Stephens, J. Novice and experienced physical therapist clinicians: a comparison of how reflection is used to inform the clinical decision-making process. Phys. Ther., 90(1):75$88,2010$.

\section{Dirección para correspondencia: \\ Rodrigo Muñoz Cofré \\ Avenida Francisco Salazar \# 01145 \\ Universidad de La Frontera \\ Temuco - CHILE}

Email: b.munoz13@ufromail.cl

Recibido : 04-06-2018

Aceptado: 27-09-2018 